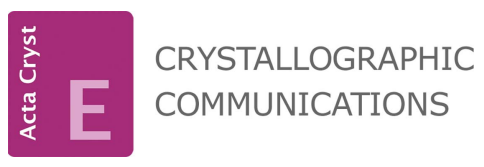

ISSN 2056-9890

Received 2 April 2019

Accepted 11 April 2019

Edited by C. Rizzoli, Universita degli Studi di Parma, Italy

Keywords: crystal structure; charge assisted hydrogen bonding; thiazolidine ring; disorder; Hirshfeld surface analysis..

CCDC reference: 1909594

Supporting information: this article has supporting information at journals.iucr.org/e

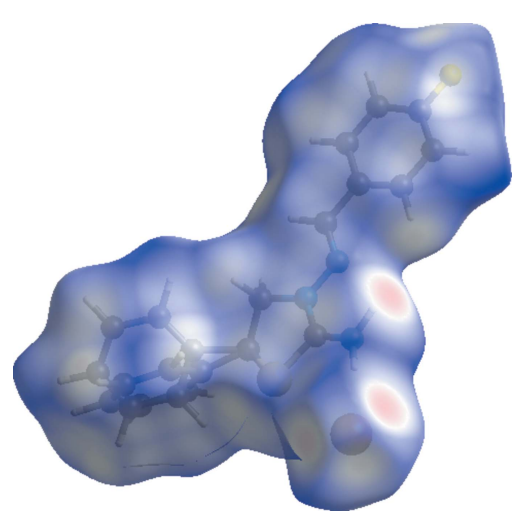

\section{Crystal structure and Hirshfeld surface analysis of (E)-3-[(4-fluorobenzylidene)amino]-5-phenylthia- zolidin-2-iminium bromide}

\author{
Ali N. Khalilov, ${ }^{\mathrm{a}, \mathrm{b}}$ Zeliha Atioğlu, ${ }^{\mathrm{c}}$ Mehmet Akkurt, ${ }^{\mathrm{d}}$ Gulnara Sh. Duruskari, ${ }^{\mathrm{a}}$ \\ Flavien A. A. Toze ${ }^{e_{*}}$ and Afet T. Huseynova ${ }^{a}$
}

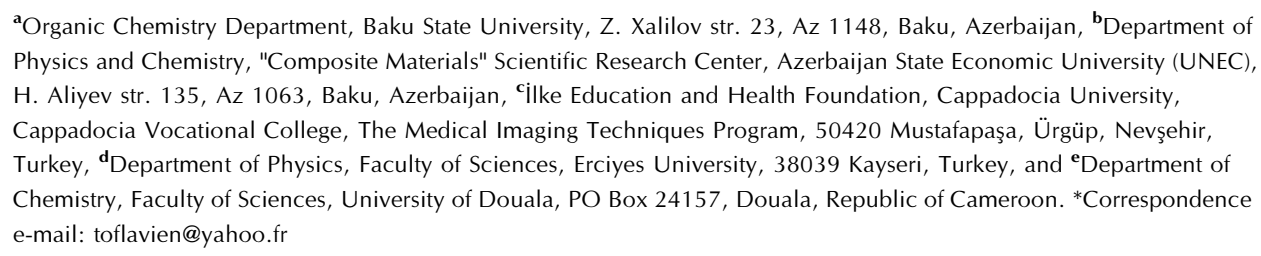

In the cation of the title salt, $\mathrm{C}_{16} \mathrm{H}_{15} \mathrm{FN}_{3} \mathrm{~S}^{+} \cdot \mathrm{Br}^{-}$, the phenyl ring is disordered over two sets of sites with a refined occupancy ratio of 0.503 (4):0.497 (4). The mean plane of the thiazolidine ring makes dihedral angles of 13.51 (14), 48.6 (3) and $76.5(3)^{\circ}$ with the fluorophenyl ring and the major- and minor-disorder components of the phenyl ring, respectively. The central thiazolidine ring adopts an envelope conformation. In the crystal, centrosymmetrically related cations and anions are linked into dimeric units via $\mathrm{N}-\mathrm{H} \cdots \mathrm{Br}$ hydrogen bonds, which are further connected by weak $\mathrm{C}-\mathrm{H} \cdots \mathrm{Br}$ hydrogen bonds into chains parallel to [110]. Hirshfeld surface analysis and two-dimensional fingerprint plots indicate that the most important contributions to the crystal packing are from $\mathrm{H} \cdots \mathrm{H}(44.3 \%), \mathrm{Br} \cdots \mathrm{H} / \mathrm{H} \cdots \mathrm{Br}(16.8 \%), \mathrm{C} \cdots \mathrm{H} / \mathrm{H} \cdots \mathrm{C}(13.9 \%), \mathrm{F} \cdots \mathrm{H} / \mathrm{H} \cdots \mathrm{F}$ $(10.3 \%)$ and $\mathrm{S} \cdots \mathrm{H} / \mathrm{H} \cdots \mathrm{S}(3.8 \%)$ interactions.

\section{Chemical context}

Noncovalent interactions, both intermolecular and intramolecular, occur in virtually every substance and play an important role in the synthesis, catalysis, design of materials and biological processes (Akbari et al., 2017; Gurbanov et al., 2018; Kopylovich et al., 2011; Maharramov et al., 2010; Mahmoudi et al., 2018a,b,c; Mahmudov et al., 2011, 2013, 2014a,b, 2015, 2017a,b, 2019; Shixaliyev et al., 2013, 2018). On the other hand, Schiff bases and related hydrazone ligands and their complexes have attracted attention over the past decades due to their potential biological, pharmacological and analytical applications (Kopylovich et al., 2011; Mahmoudi et al., 2018a,b,c; Mahmudov et al., 2013). Hetercyclic amines are also widely used in the synthesis of Schiff bases, which provide different kinds of noncovalent interactions. As a further study in this field, we report herein the crystal structure and Hirshfeld surface analysis of the title compound.

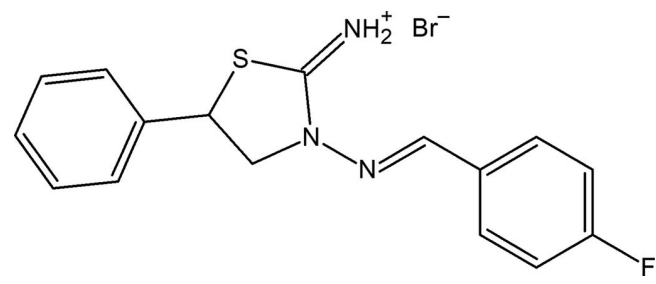


Table 1

Hydrogen-bond geometry $\left(\AA \stackrel{\circ}{\circ}^{\circ}\right)$.

\begin{tabular}{lllll}
\hline$D-\mathrm{H} \cdots A$ & $D-\mathrm{H}$ & $\mathrm{H} \cdots A$ & $D \cdots A$ & $D-\mathrm{H} \cdots A$ \\
\hline $\mathrm{N} 3-\mathrm{H} 3 A \cdots \mathrm{Br} 1$ & 0.90 & 2.36 & $3.2557(18)$ & 172 \\
$\mathrm{~N} 3-\mathrm{H} 3 B \cdots \mathrm{Br} 1^{\mathrm{i}}$ & 0.90 & 2.55 & $3.3552(18)$ & 150 \\
$\mathrm{C} 4-\mathrm{H} 4 A \cdots \mathrm{Br}^{\mathrm{ii}}$ & 0.93 & 2.99 & $3.726(2)$ & 137
\end{tabular}

Symmetry codes: (i) $-x+1,-y,-z+1$; (ii) $x+1, y+1, z$.

\section{Structural commentary}

The thiazolidine ring $(\mathrm{S} 1 / \mathrm{N} 2 / \mathrm{C} 1-\mathrm{C} 3)$ in the cation of the title salt (Fig. 1) adopts an envelope conformation, with puckering parameters of $Q(2)=0.321$ (3) $\AA$ and $\varphi(2)=43.3(5)^{\circ}$. The mean plane of the thiazolidine ring makes dihedral angles of 13.51 (14), $48.6(3)$ and $76.5(3)^{\circ}$ with the fluorophenyl ring (C5-C10) and the major- and minor-disorder components (C11-C16 and $\left.\mathrm{C} 11^{\prime}-\mathrm{C} 16^{\prime}\right)$ of the phenyl ring, respectively. The $\mathrm{N} 2-\mathrm{N} 1-\mathrm{C} 4-\mathrm{C} 5$ bridge that links the thiazolidine and 4fluorophenyl rings has a torsion angle of $-177.36(19)^{\circ}$.

\section{Supramolecular features and Hirshfeld surface analysis}

In the crystal, centrosymmetrically related cations and anions are linked via pairs of $\mathrm{N}-\mathrm{H} \cdots \mathrm{Br}$ hydrogen bonds (Table 1 ) into dimeric units forming rings of $R_{4}^{2}(8)$ graph-set motif (Fig. 2). The dimers are further connected by weak $\mathrm{C}-\mathrm{H} \cdots \mathrm{Br}$ interactions to form chains running parallel to [110].

Hirshfeld surface analysis was used to investigate the presence of hydrogen bonds and intermolecular interactions in the crystal structure. The Hirshfeld surface analysis (Spackman \& Jayatilaka, 2009) of the title salt was generated by CrystalExplorer3.1 (Wolff et al., 2012), and comprised $d_{\text {norm }}$ surface plots and 2D fingerprint plots (Spackman \&

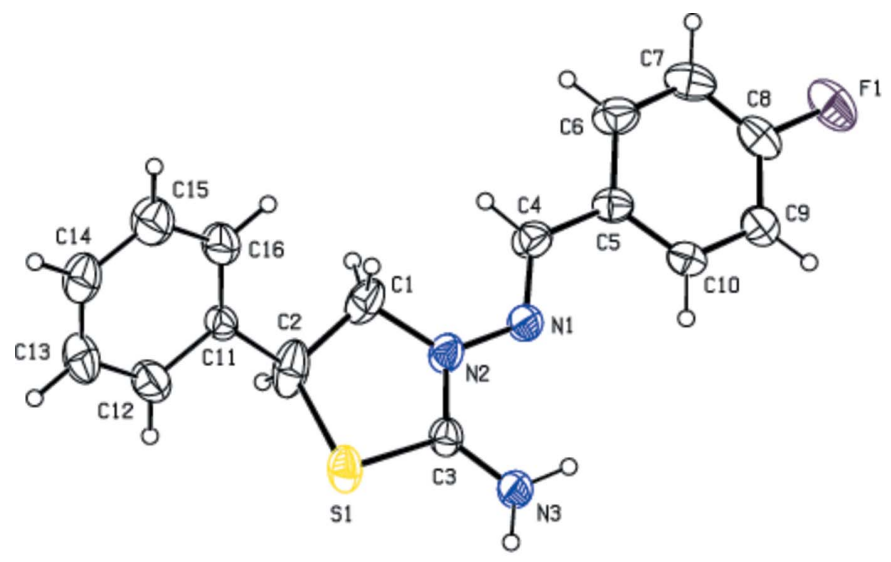

Figure 1 Br1

The molecular structure of the title salt. Displacement ellipsoids are drawn at the $30 \%$ probability level. $\mathrm{H}$ atoms are shown as spheres of arbitrary radius. The minor-disorder component has been omitted for clarity

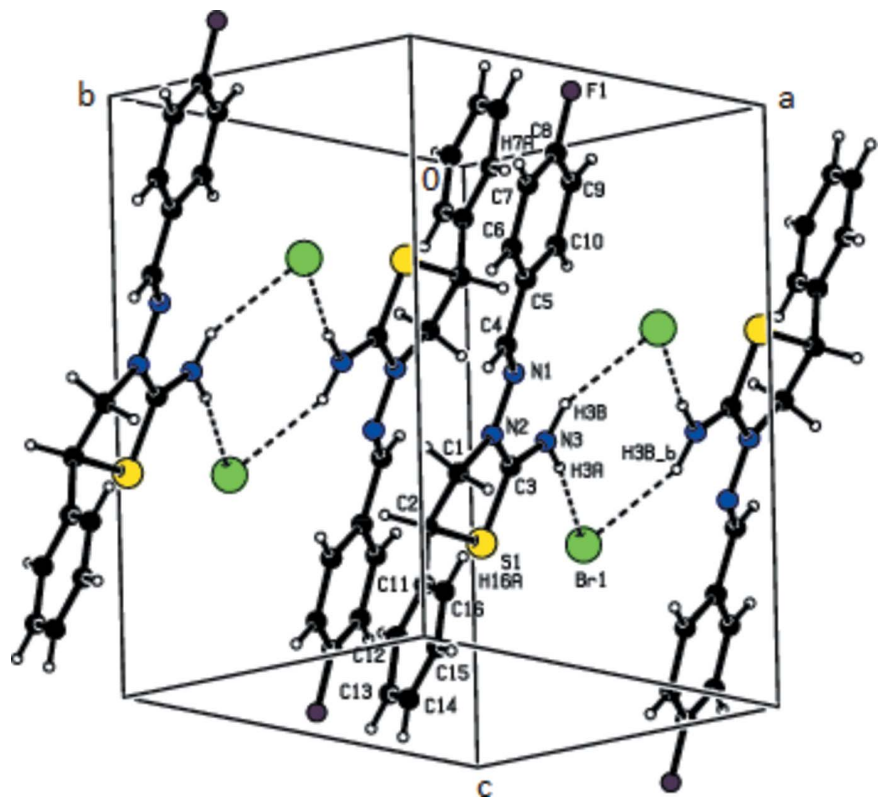

Figure 2

A view of the intermolecular $\mathrm{N}-\mathrm{H} \cdots \mathrm{Br}$ hydrogen bonds of the title salt in the unit cell. The minor-disorder component has been omitted for clarity

McKinnon, 2002). The plots of the Hirshfeld surface mapped over $d_{\text {norm }}$ using a standard surface resolution with a fixed colour scale of -1.4747 (red) to 1.2166 a.u. (blue) is shown in Fig. 3. This plot was generated to quantify and visualize the intermolecular interactions and to explain the observed crystal packing.

The shape index of the Hirshfeld surface is a tool to visualize $\pi-\pi$ stacking interactions by the presence of adjacent red and blue triangles; if there are no adjacent red and/or blue

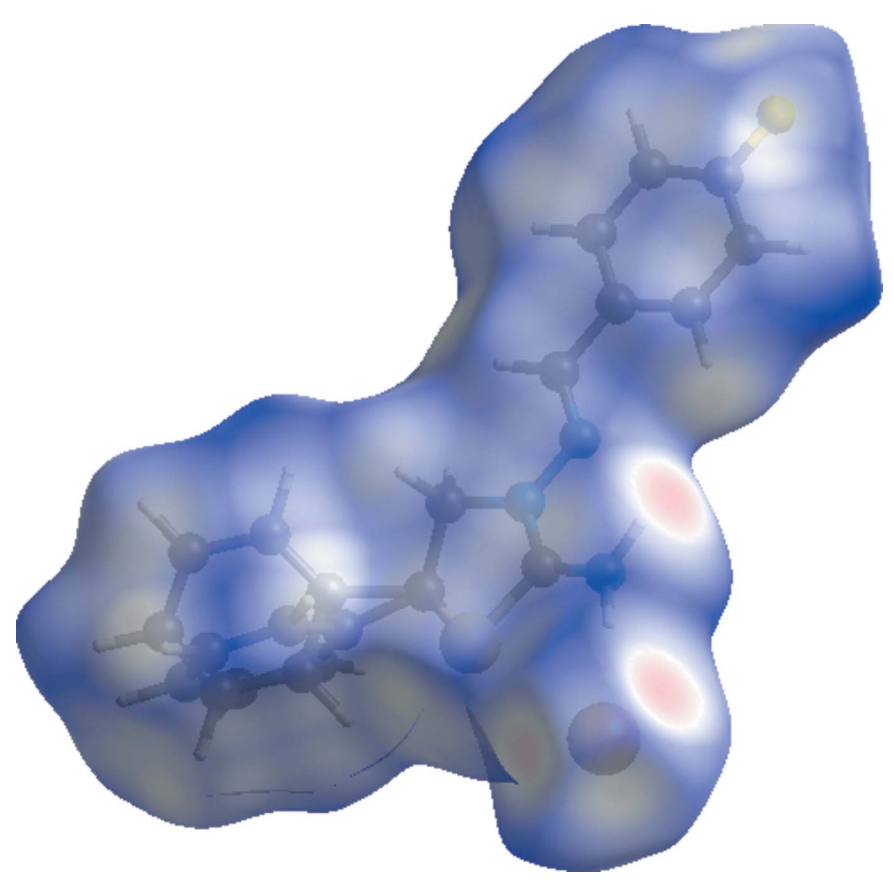

Figure 3

Hirshfeld surface of the title salt mapped with $d_{\text {norm }}$. 


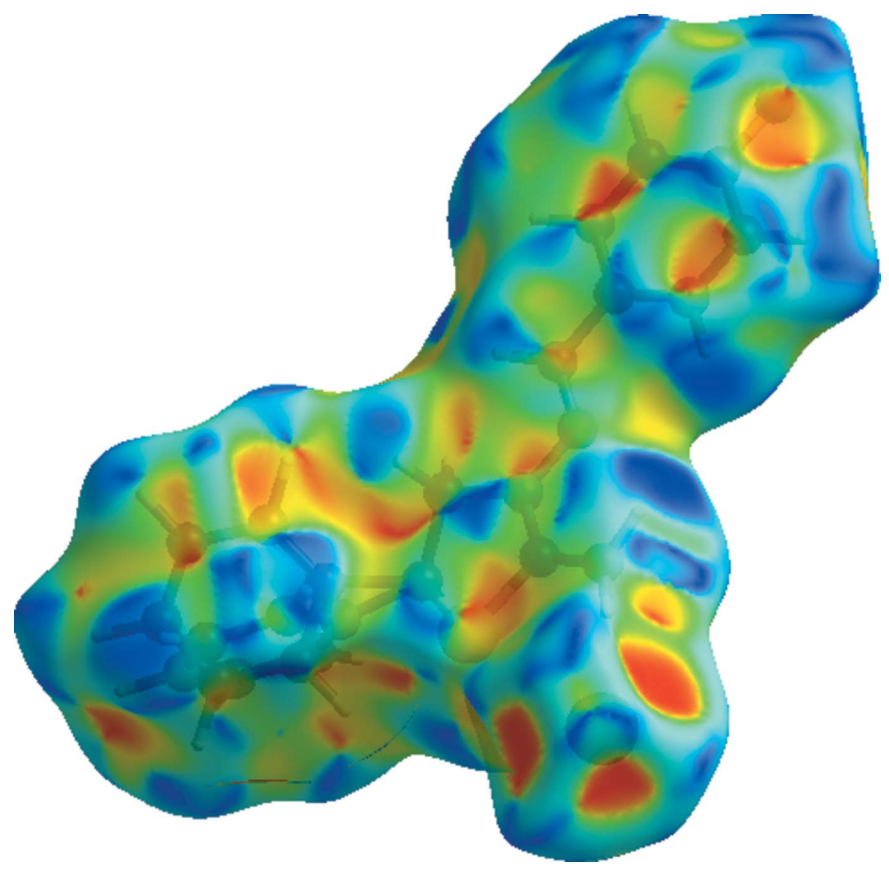

Figure 4

Hirshfeld surface of the title salt mapped with shape index.
Table 2

Percentage contributions of interatomic contacts to the Hirshfeld surface for the title salt.

\begin{tabular}{ll}
\hline Contact & Percentage contribution \\
\hline $\mathrm{H} \cdots \mathrm{H}$ & 44.3 \\
$\mathrm{Br} \cdots \mathrm{H} / \mathrm{H} \cdots \mathrm{Br}$ & 16.8 \\
$\mathrm{C} \cdots \mathrm{H} / \mathrm{H} \cdots \mathrm{C}$ & 13.9 \\
$\mathrm{~F} \cdots \mathrm{H} / \mathrm{H} \cdots \mathrm{F}$ & 10.3 \\
$\mathrm{~S} \cdots \mathrm{H} / \mathrm{H} \cdots \mathrm{S}$ & 3.8 \\
$\mathrm{~N} \cdots \mathrm{C} / \mathrm{C} \cdots \mathrm{N}$ & 3.6 \\
$\mathrm{~S} \cdots \mathrm{C} / \mathrm{C} \cdots \mathrm{S}$ & 2.7 \\
$\mathrm{~N} \cdots \mathrm{H} / \mathrm{H} \cdots \mathrm{N}$ & 1.8 \\
$\mathrm{C} \cdots \mathrm{C}$ & 1.5 \\
$\mathrm{~N} \cdots \mathrm{N}$ & 0.7 \\
$\mathrm{Br} \cdots \mathrm{C} / \mathrm{C} \cdots \mathrm{Br}$ & 0.3 \\
$\mathrm{~S} \cdots \mathrm{N} / \mathrm{N} \cdots \mathrm{S}$ & 0.3 \\
$\mathrm{~F} \cdots \mathrm{C} / \mathrm{C} \cdots \mathrm{F}$ & 0.2
\end{tabular}

triangles, then there are no $\pi-\pi$ interactions. Fig. 4 clearly suggest that there are no $\pi-\pi$ interactions present in the title salt.

Fig. 5(a) shows the 2D fingerprint of the sum of the contacts contributing to the Hirshfeld surface represented in normal mode. These represent both the overall 2D fingerprint plots
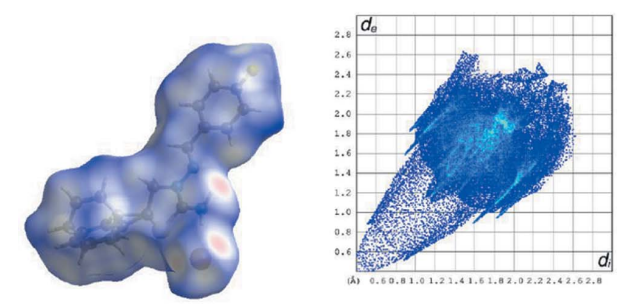

(a) All...All
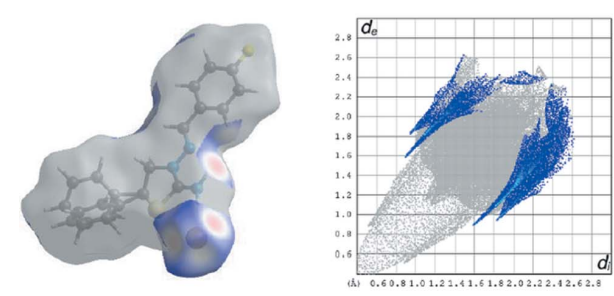

(c) Br...H / H...Br
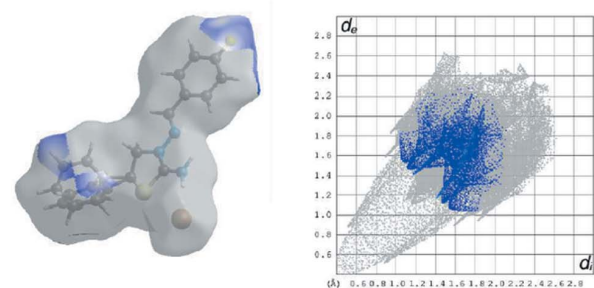

(e) F...H / H...F
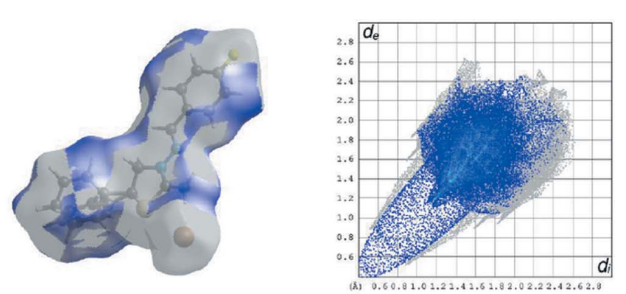

(b) H...H
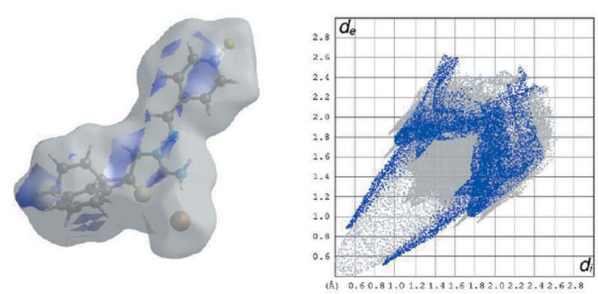

(d) C...H / H...C
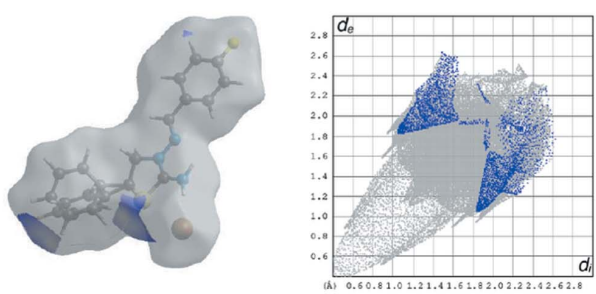

(f) S...H / H...S

Figure 5

The 2D fingerprint plots of the title salt, showing $(a)$ all interactions, and delineated into $(b) \mathrm{H} \cdots \mathrm{H},(c) \mathrm{Br} \cdots \mathrm{H} / \mathrm{H} \cdots \mathrm{Br},(d) \mathrm{C} \cdots \mathrm{H} / \mathrm{H} \cdots \mathrm{C},(e) \mathrm{F} \cdots \mathrm{H} /$ $\mathrm{H} \cdots \mathrm{F}$ and $(f) \mathrm{S} \cdots \mathrm{H} / \mathrm{H} \cdots \mathrm{S}$ interactions $\left[d_{\mathrm{e}}\right.$ and $d_{\mathrm{i}}$ represent the distances from a point on the Hirshfeld surface to the nearest atoms outside (external) and inside (internal) the surface, respectively]. 
and those that represent $\mathrm{H} \cdots \mathrm{H}(44.3 \%), \mathrm{Br} \cdots \mathrm{H} / \mathrm{H} \cdots \mathrm{Br}$ $(16.8 \%), \mathrm{C} \cdots \mathrm{H} / \mathrm{H} \cdots \mathrm{C}(13.9 \%), \mathrm{F} \cdots \mathrm{H} / \mathrm{H} \cdots \mathrm{F}(10.3 \%)$ and $\mathrm{S} \cdots \mathrm{H} / \mathrm{H} \cdots \mathrm{S}(3.8 \%)$ contacts, respectively (Figs. $5 b-f)$. The most significant intermolecular interactions are the $\mathrm{H} \cdots \mathrm{H}$ interactions $(44.3 \%)$ (Fig. $6 b$ ). All the contributions to the Hirshfeld surface are given in Table 2.

\subsection{Database survey}

A search of the Cambridge Structural Database (CSD, Version 5.40, update of November 2018; Groom et al., 2016) for 2-thiazolidiniminium compounds gave seven hits, viz. UDELUN (Akkurt et al., 2018), WILBIC (Marthi et al., 1994), WILBOI (Marthi et al., 1994), WILBOI01 (Marthi et al., 1994), YITCEJ (Martem'yanova et al., 1993a), YITCAF (Martem'yanova et al., 1993b) and YOPLUK (Marthi et al., 1995).

In the crystal of UDELUN (Akkurt et al., 2018), $\mathrm{C}-\mathrm{H} \cdots \mathrm{Br}$ and $\mathrm{N}-\mathrm{H} \cdots \mathrm{Br}$ hydrogen bonds link the components into a three-dimensional network with the cations and anions stacked along the $b$-axis direction. Weak $\mathrm{C}-\mathrm{H} \cdots \pi$ interactions, which only involve the minor-disorder component of the ring, also contribute to the molecular packing. In addition, there are also inversion-related $\mathrm{Cl} \cdots \mathrm{Cl}$ halogen bonds and $\mathrm{C}-\mathrm{Cl} \cdots \pi$ (ring) contacts.

In the remaining structures, the $3-\mathrm{N}$ atom carries a Csubstituent instead of an $\mathrm{N}$-substituent, as found in the title compound. The first three crystal structures were determined for racemic (WILBIC; Marthi et al., 1994) and two optically active samples (WILBOI and WILBOI01; Marthi et al., 1994) of 3-(2'-chloro-2'-phenylethyl)-2-thiazolidiniminium $p$-toluenesulfonate. In all three structures, the most disordered fragment of these molecules is the asymmetric $\mathrm{C}$ atom and the $\mathrm{Cl}$ atom attached to it. The disorder of the cation in the racemate corresponds to the presence of both enantiomers at each site in the ratio 0.821(3):0.179(3). The system of hydrogen bonds connecting two cations and two anions into 12 -membered rings is identical in the racemic and in the optically active crystals. YITCEJ (Martem'yanova et al., 1993a) is a product of the interaction of 2-amino-5-methylthiazoline with methyl iodide, with alkylation at the endocylic $\mathrm{N}$ atom, while YITCAF (Martem'yanova et al., 1993b) is a product of the reaction of 3-nitro-5-methoxy-, 3-nitro-5chloro- and 3-bromo-5-nitrosalicylaldehyde with the heterocyclic base to form the salt-like complexes.

\section{Synthesis and crystallization}

To a solution of 3-amino-5-phenylthiazolidin-2-iminium bromide $(1 \mathrm{mmol})$ in ethanol $(20 \mathrm{ml})$ was added 4 -fluorobenzaldehyde $(1 \mathrm{mmol})$. The mixture was refluxed for $2 \mathrm{~h}$ and then cooled. The reaction product precipitated from the reaction mixture as colourless single crystals, was collected by filtration and washed with cold acetone (yield 64\%; m.p. 544-545 K). Analysis calculated (\%) for $\mathrm{C}_{16} \mathrm{H}_{15} \mathrm{BrFN}_{3} \mathrm{~S}$ : C 50.53, H 3.98, N 11.05; found: C 50.47, H 3.93, N 11.00. ${ }^{1} \mathrm{H}$ NMR $(300 \mathrm{MHz}$, DMSO- $\left.d_{6}\right): 4.56\left(k, 1 \mathrm{H}, \mathrm{CH}_{2},{ }^{3} J_{\mathrm{H}-\mathrm{H}}=6.6 \mathrm{~Hz}\right), 4,87\left(t, 1 \mathrm{H}, \mathrm{CH}_{2}\right.$,
Table 3

Experimental details.

\begin{tabular}{|c|c|}
\hline \multicolumn{2}{|l|}{ Crystal data } \\
\hline Chemical formula & $\mathrm{C}_{16} \mathrm{H}_{15} \mathrm{FN}_{3} \mathrm{~S}^{+} \cdot \mathrm{Br}^{-}$ \\
\hline$M_{\mathrm{r}}$ & 380.28 \\
\hline Crystal system, space group & Triclinic, $P \overline{1}$ \\
\hline Temperature $(\mathrm{K})$ & 296 \\
\hline$a, b, c(\AA)$ & $8.0599(3), 8.6086(4), 12.7608(5)$ \\
\hline$\alpha, \beta, \gamma\left({ }^{\circ}\right)$ & $96.548(2), 92.518(2), 111.065(2)$ \\
\hline$V\left(\AA^{3}\right)$ & $817.39(6)$ \\
\hline$Z$ & 2 \\
\hline Radiation type & Мо $K \alpha$ \\
\hline$\mu\left(\mathrm{mm}^{-1}\right)$ & 2.65 \\
\hline Crystal size $(\mathrm{mm})$ & $0.16 \times 0.12 \times 0.09$ \\
\hline \multicolumn{2}{|l|}{ Data collection } \\
\hline Diffractometer & Bruker APEXII CCD \\
\hline Absorption correction & $\begin{array}{l}\text { Multi-scan (SADABS; Bruker, } \\
\text { 2003) }\end{array}$ \\
\hline$T_{\min }, T_{\max }$ & $0.664,0.782$ \\
\hline $\begin{array}{l}\text { No. of measured, independent and } \\
\text { observed }[I>2 \sigma(I)] \text { reflections }\end{array}$ & $12009,3321,2672$ \\
\hline$R_{\text {int }}$ & 0.025 \\
\hline$(\sin \theta / \lambda)_{\max }\left(\AA^{-1}\right)$ & 0.627 \\
\hline \multicolumn{2}{|l|}{ Refinement } \\
\hline$R\left[F^{2}>2 \sigma\left(F^{2}\right)\right], w R\left(F^{2}\right), S$ & $0.029,0.077,1.04$ \\
\hline No. of reflections & 3321 \\
\hline No. of parameters & 254 \\
\hline $\mathrm{H}$-atom treatment & $\mathrm{H}$-atom parameters constrained \\
\hline$\Delta \rho_{\max }, \Delta \rho_{\min }\left(\mathrm{e} \AA^{-3}\right)$ & $0.37,-0.48$ \\
\hline
\end{tabular}

Computer programs: APEX2 (Bruker, 2007), SAINT (Bruker, 2007), SHELXS97 (Sheldrick, 2008), SHELXL2014 (Sheldrick, 2015), ORTEP-3 for Windows (Farrugia, 2012) and PLATON (Spek, 2003).

$\left.{ }^{3} J_{\mathrm{H}-\mathrm{H}}=7.8 \mathrm{~Hz}\right), 5.60\left(t, 1 \mathrm{H}, \mathrm{CH}-\mathrm{Ar}, 3 J_{\mathrm{H}-\mathrm{H}}=7.8 \mathrm{~Hz}\right), 7.32-8.16$ $(m, 9 \mathrm{H}, 9 \mathrm{Ar}-\mathrm{H}), 8.45(s, 1 \mathrm{H}, \mathrm{CH}=), 10.37\left(s, 2 \mathrm{H}, \mathrm{NH}_{2}\right) \cdot{ }^{13} \mathrm{C}$ NMR $\left(75 \mathrm{MHz}\right.$, DMSO- $\left.d_{6}\right)$ : 45.39, 55.97, $116.05,127.81$, 128.91, 129.13, 129.60, 131.05, 131.17, 137.55, 150.00, 167.89. MS (ESI), $m / z: 300.36\left[\mathrm{C}_{16} \mathrm{H}_{15} \mathrm{FN}_{3} \mathrm{~S}\right]^{+}$and $79.88 \mathrm{Br}^{-}$.

\section{Refinement details}

Crystal data, data collection and structure refinement details are summarized in Table 3. All $\mathrm{H}$ atoms were positioned geometrically and refined using a riding model, with $\mathrm{N}-\mathrm{H}=$ $0.90 \AA$ and $\mathrm{C}-\mathrm{H}=0.93-0.98 \AA$, and with $U_{\text {iso }}(\mathrm{H})=$ $1.2 U_{\mathrm{eq}}(\mathrm{C}, \mathrm{N})$. The phenyl ring in the cation is disordered over two sets of sites with an occupancy ratio of 0.503 (4):0.497 (4). Seven outliers $(001 ; \overline{3} 05 ; \overline{1} 43 ; 010 ; \overline{2} 75 ; \overline{2}, \overline{1}, 12$; and $7 \overline{5} 3)$ were omitted in the final cycles of refinement.

\section{Acknowledgements}

Ali Khalilov is grateful to Baku State University for the '50+50' individual grant in support of this work.

\section{References}

Akbari Afkhami, F., Mahmoudi, G., Gurbanov, A. V., Zubkov, F. I., Qu, F., Gupta, A. \& Safin, D. A. (2017). Dalton Trans. 46, 1488814896.

Akkurt, M., Duruskari, G. S., Toze, F. A. A., Khalilov, A. N. \& Huseynova, A. T. (2018). Acta Cryst. E74, 1168-1172.

Bruker (2003). SADABS. Bruker AXS Inc., Madison, Wisconsin, USA. 
Bruker (2007). APEX2 and SAINT. Bruker AXS Inc., Madison, Wisconsin, USA.

Farrugia, L. J. (2012). J. Appl. Cryst. 45, 849-854.

Groom, C. R., Bruno, I. J., Lightfoot, M. P. \& Ward, S. C. (2016). Acta Cryst. B72, 171-179.

Gurbanov, A. V., Maharramov, A. M., Zubkov, F. I., Saifutdinov, A. M. \& Guseinov, F. I. (2018). Aust. J. Chem. 71, 190-194.

Kopylovich, M. N., Mahmudov, K. T., Haukka, M., Luzyanin, K. V. \& Pombeiro, A. J. L. (2011). Inorg. Chim. Acta, 374, 175-180.

Maharramov, A. M., Aliyeva, R. A., Aliyev, I. A., Pashaev, F. G., Gasanov, A. G., Azimova, S. I., Askerov, R. K., Kurbanov, A. V. \& Mahmudov, K. T. (2010). Dyes Pigments, 85, 1-6.

Mahmoudi, G., Seth, S. K., Bauzá, A., Zubkov, F. I., Gurbanov, A. V., White, J., Stilinović, V., Doert, Th. \& Frontera, A. (2018a). CrystEngComm, 20, 2812-2821.

Mahmoudi, G., Zangrando, E., Mitoraj, M. P., Gurbanov, A. V., Zubkov, F. I., Moosavifar, M., Konyaeva, I. A., Kirillov, A. M. \& Safin, D. A. (2018b). New J. Chem. 42, 4959-4971.

Mahmoudi, G., Zaręba, J. K., Gurbanov, A. V., Bauzá, A., Zubkov, F. I., Kubicki, M., Stilinović, V., Kinzhybalo, V. \& Frontera, A. (2018c). Eur. J. Inorg. Chem. pp. 4763-4772.

Mahmudov, K. T., Guedes da Silva, M. F. C., Kopylovich, M. N., Fernandes, A. R., Silva, A., Mizar, A. \& Pombeiro, A. J. L. (2014a). J. Organomet. Chem. 760, 67-73.

Mahmudov, K. T., Guedes da Silva, M. F. C., Sutradhar, M., Kopylovich, M. N., Huseynov, F. E., Shamilov, N. T., Voronina, A. A., Buslaeva, T. M. \& Pombeiro, A. J. L. (2015). Dalton Trans. 44, 5602-5610.

Mahmudov, K. T., Gurbanov, A. V., Guseinov, F. I. \& Guedes da Silva, M. F. C. (2019). Coord. Chem. Rev. 387, 32-46.

Mahmudov, K. T., Kopylovich, M. N., Guedes da Silva, M. F. C. \& Pombeiro, A. J. L. (2017a). Coord. Chem. Rev. 345, 54-72.

Mahmudov, K. T., Kopylovich, M. N., Guedes da Silva, M. F. C. \& Pombeiro, A. J. L. (2017b). Dalton Trans. 46, 10121-10138.
Mahmudov, K. T., Kopylovich, M. N., Maharramov, A. M., Kurbanova, M. M., Gurbanov, A. V. \& Pombeiro, A. J. L. (2014b). Coord. Chem. Rev. 265, 1-37.

Mahmudov, K. T., Kopylovich, M. N. \& Pombeiro, A. J. L. (2013). Coord. Chem. Rev. 257, 1244-1281.

Mahmudov, K. T., Maharramov, A. M., Aliyeva, R. A., Aliyev, I. A., Askerov, R. K., Batmaz, R., Kopylovich, M. N. \& Pombeiro, A. J. L. (2011). J. Photochem. Photobiol. Chem. 219, 159-165.

Martem'yanova, N. A., Chunaev, Y. M., Przhiyalgovskaya, N. M., Kurkovskaya, L. N., Filipenko, O. S. \& Aldoshin, S. M. (1993a). Khim. Geterotsikl. Soedin. pp. 415-419.

Martem'yanova, N. A., Chunaev, Y. M., Przhiyalgovskaya, N. M., Kurkovskaya, L. N., Filipenko, O. S. \& Aldoshin, S. M. (1993b). Khim. Geterotsikl. Soedin. pp. 420-425.

Marthi, K., Larsen, M., Ács, M., Bálint, J. \& Fogassy, E. (1995). Acta Chem. Scand. 49, 20-27.

Marthi, K., Larsen, S., Ács, M., Bálint, J. \& Fogassy, E. (1994). Acta Cryst. B50, 762-771.

Sheldrick, G. M. (2008). Acta Cryst. A64, 112-122.

Sheldrick, G. M. (2015). Acta Cryst. C71, 3-8.

Shixaliyev, N. Q., Ahmadova, N. E., Gurbanov, A. V., Maharramov, A. M., Mammadova, G. Z., Nenajdenko, V. G., Zubkov, F. I., Mahmudov, K. T. \& Pombeiro, A. J. L. (2018). Dyes Pigments, 150, 377-381.

Shixaliyev, N. Q., Maharramov, A. M., Gurbanov, A. V., Nenajdenko, V. G., Muzalevskiy, V. M., Mahmudov, K. T. \& Kopylovich, M. N. (2013). Catal. Today, 217, 76-79.

Spackman, M. A. \& Jayatilaka, D. (2009). CrystEngComm, 11, 19-32.

Spackman, M. A. \& McKinnon, J. J. (2002). CrystEngComm, 4, 378392.

Spek, A. L. (2003). J. Appl. Cryst. 36, 7-13.

Wolff, S. K., Grimwood, D. J., McKinnon, J. J., Turner, M. J., Jayatilaka, D. \& Spackman, M. A. (2012). CrystalExplorer. University of Western Australia. 


\section{supporting information}

Acta Cryst. (2019). E75, 662-666 [https://doi.org/10.1107/S2056989019004973]

Crystal structure and Hirshfeld surface analysis of $(E)-3$-[(4-fluorobenzylidene)amino]-5-phenylthiazolidin-2-iminium bromide

Ali N. Khalilov, Zeliha Atioğlu, Mehmet Akkurt, Gulnara Sh. Duruskari, Flavien A. A. Toze and Afet T. Huseynova

Computing details

Data collection: APEX2 (Bruker, 2007); cell refinement: SAINT (Bruker, 2007); data reduction: SAINT (Bruker, 2007); program(s) used to solve structure: SHELXS97 (Sheldrick, 2008); program(s) used to refine structure: SHELXL2014 (Sheldrick, 2015); molecular graphics: ORTEP-3 for Windows (Farrugia, 2012); software used to prepare material for publication: PLATON (Spek, 2003).

(E)-3-[(4-Fluorobenzylidene)amino]-5-phenylthiazolidin-2-iminium bromide

Crystal data

$\mathrm{C}_{16} \mathrm{H}_{15} \mathrm{FN}_{3} \mathrm{~S}^{+} \cdot \mathrm{Br}^{-}$

$M_{r}=380.28$

Triclinic, $P \overline{1}$

$a=8.0599$ (3) $\AA$

$b=8.6086(4) \AA$

$c=12.7608(5) \AA$

$\alpha=96.548(2)^{\circ}$

$\beta=92.518(2)^{\circ}$

$\gamma=111.065(2)^{\circ}$

$V=817.39(6) \AA^{3}$

Data collection

Bruker APEXII CCD

diffractometer

$\varphi$ and $\omega$ scans

Absorption correction: multi-scan

(SADABS; Bruker, 2003)

$T_{\min }=0.664, T_{\max }=0.782$

12009 measured reflections

Refinement

Refinement on $F^{2}$

Least-squares matrix: full

$R\left[F^{2}>2 \sigma\left(F^{2}\right)\right]=0.029$

$w R\left(F^{2}\right)=0.077$

$S=1.03$

3321 reflections

254 parameters

0 restraints
$Z=2$

$F(000)=384$

$D_{\mathrm{x}}=1.545 \mathrm{Mg} \mathrm{m}^{-3}$

Mo $K \alpha$ radiation, $\lambda=0.71073 \AA$

Cell parameters from 5655 reflections

$\theta=2.6-26.3^{\circ}$

$\mu=2.65 \mathrm{~mm}^{-1}$

$T=296 \mathrm{~K}$

Plate, colorless

$0.16 \times 0.12 \times 0.09 \mathrm{~mm}$

3321 independent reflections

2672 reflections with $I>2 \sigma(I)$

$R_{\text {int }}=0.025$

$\theta_{\text {max }}=26.5^{\circ}, \theta_{\min }=2.7^{\circ}$

$h=-10 \rightarrow 7$

$k=-10 \rightarrow 10$

$l=-14 \rightarrow 15$

Hydrogen site location: mixed

$\mathrm{H}$-atom parameters constrained

$w=1 /\left[\sigma^{2}\left(F_{\mathrm{o}}^{2}\right)+(0.0376 P)^{2}+0.2228 P\right]$

where $P=\left(F_{\mathrm{o}}^{2}+2 F_{\mathrm{c}}^{2}\right) / 3$

$(\Delta / \sigma)_{\max }=0.001$

$\Delta \rho_{\max }=0.37 \mathrm{e} \AA^{-3}$

$\Delta \rho_{\min }=-0.48$ e $\AA^{-3}$ 


\section{Special details}

Geometry. All esds (except the esd in the dihedral angle between two 1.s. planes) are estimated using the full covariance matrix. The cell esds are taken into account individually in the estimation of esds in distances, angles and torsion angles; correlations between esds in cell parameters are only used when they are defined by crystal symmetry. An approximate (isotropic) treatment of cell esds is used for estimating esds involving l.s. planes.

Fractional atomic coordinates and isotropic or equivalent isotropic displacement parameters $\left(\AA^{2}\right)$

\begin{tabular}{|c|c|c|c|c|c|}
\hline & $x$ & $y$ & $z$ & $U_{\text {iso }} * / U_{\text {eq }}$ & Occ. $(<1)$ \\
\hline $\mathrm{Br} 1$ & $0.28837(4)$ & $-0.06846(3)$ & $0.65093(2)$ & $0.07274(12)$ & \\
\hline S1 & $0.50535(9)$ & $0.40641(8)$ & $0.72511(4)$ & $0.06317(17)$ & \\
\hline $\mathrm{F} 1$ & $1.1652(2)$ & $0.6889(3)$ & $0.07197(13)$ & 0.0994 (6) & \\
\hline N1 & $0.7677(2)$ & $0.5288(2)$ & 0.48291 (13) & $0.0510(4)$ & \\
\hline $\mathrm{C} 1$ & $0.7491(4)$ & $0.6768(3)$ & $0.66453(19)$ & $0.0676(7)$ & \\
\hline $\mathrm{H} 1 \mathrm{~A}$ & 0.765957 & 0.780305 & 0.635640 & $0.081 *$ & \\
\hline H1B & 0.859205 & 0.688530 & 0.704392 & $0.081 *$ & \\
\hline $\mathrm{N} 2$ & $0.6981(3)$ & $0.5342(2)$ & $0.57951(13)$ & $0.0540(4)$ & \\
\hline $\mathrm{C} 2$ & $0.5974(5)$ & $0.6381(4)$ & $0.73431(19)$ & $0.0803(9)$ & \\
\hline $\mathrm{H} 2 \mathrm{~A}$ & 0.506784 & 0.680819 & 0.710523 & $0.096^{*}$ & \\
\hline N3 & $0.5389(3)$ & $0.2468(2)$ & 0.54077 (14) & $0.0564(5)$ & \\
\hline $\mathrm{H} 3 \mathrm{~A}$ & 0.472588 & 0.153018 & 0.566419 & $0.068^{*}$ & \\
\hline H3B & 0.598218 & 0.240158 & 0.483319 & $0.068 *$ & \\
\hline C3 & $0.5851(3)$ & $0.3881(3)$ & $0.60334(16)$ & $0.0489(5)$ & \\
\hline $\mathrm{C} 4$ & $0.8962(3)$ & $0.6588(3)$ & $0.46468(18)$ & $0.0583(6)$ & \\
\hline $\mathrm{H} 4 \mathrm{~A}$ & 0.943400 & 0.751707 & 0.516854 & $0.070^{*}$ & \\
\hline $\mathrm{C} 5$ & $0.9692(3)$ & $0.6619(3)$ & $0.36204(18)$ & $0.0532(5)$ & \\
\hline C6 & $1.0980(3)$ & $0.8092(3)$ & $0.3405(2)$ & $0.0691(7)$ & \\
\hline H6A & 1.139096 & 0.902914 & 0.392330 & $0.083^{*}$ & \\
\hline $\mathrm{C} 7$ & $1.1660(4)$ & $0.8188(4)$ & $0.2431(2)$ & $0.0767(8)$ & \\
\hline $\mathrm{H} 7 \mathrm{~A}$ & 1.254083 & 0.916970 & 0.229104 & $0.092 *$ & \\
\hline $\mathrm{C} 8$ & $1.1011(3)$ & $0.6810(4)$ & $0.1683(2)$ & $0.0665(7)$ & \\
\hline $\mathrm{C} 9$ & $0.9756(3)$ & $0.5314(3)$ & $0.1862(2)$ & $0.0659(6)$ & \\
\hline H9A & 0.935350 & 0.438646 & 0.133610 & $0.079 *$ & \\
\hline $\mathrm{C} 10$ & $0.9115(3)$ & 0.5229 & $0.28380(19)$ & $0.0592(6)$ & \\
\hline $\mathrm{H} 10 \mathrm{~A}$ & 0.827792 & 0.422230 & 0.298028 & $0.071^{*}$ & \\
\hline C11 & $0.6893(9)$ & $0.7289(7)$ & $0.8505(5)$ & $0.0447(13)$ & $0.503(4)$ \\
\hline $\mathrm{C} 12$ & $0.5575(7)$ & $0.6983(7)$ & $0.9197(5)$ & $0.0691(15)$ & $0.503(4)$ \\
\hline $\mathrm{H} 12 \mathrm{~A}$ & 0.439229 & 0.635805 & 0.894732 & $0.083^{*}$ & $0.503(4)$ \\
\hline $\mathrm{C} 13$ & $0.6019(12)$ & $0.7611(13)$ & $1.0269(6)$ & $0.074(2)$ & $0.503(4)$ \\
\hline H13A & 0.513251 & 0.739287 & 1.073481 & $0.089^{*}$ & $0.503(4)$ \\
\hline $\mathrm{C} 14$ & $0.7747(12)$ & $0.8546(10)$ & $1.0639(5)$ & $0.0740(16)$ & $0.503(4)$ \\
\hline $\mathrm{H} 14 \mathrm{~A}$ & 0.804349 & 0.898834 & 1.135130 & $0.089 *$ & 0.503 (4) \\
\hline $\mathrm{C} 15$ & $0.9041(8)$ & $0.8822(8)$ & $0.9946(4)$ & $0.0811(17)$ & $0.503(4)$ \\
\hline H15A & 1.022734 & 0.943608 & 1.019215 & $0.097 *$ & $0.503(4)$ \\
\hline $\mathrm{C} 16$ & $0.8600(7)$ & $0.8201(7)$ & $0.8893(4)$ & $0.0660(14)$ & $0.503(4)$ \\
\hline H16A & 0.949586 & 0.841027 & 0.843322 & $0.079 *$ & $0.503(4)$ \\
\hline $\mathrm{C} 11^{\prime}$ & $0.5982(9)$ & $0.7008(8)$ & $0.8494(5)$ & $0.0489(14)$ & $0.497(4)$ \\
\hline $\mathrm{C} 12^{\prime}$ & $0.5168(7)$ & $0.8137(6)$ & $0.8805(4)$ & $0.0631(13)$ & $0.497(4)$ \\
\hline
\end{tabular}




\begin{tabular}{llllll} 
H12B & 0.447047 & 0.841350 & 0.831371 & $0.076^{*}$ & $0.497(4)$ \\
C13' & $0.5408(8)$ & $0.8852(7)$ & $0.9865(5)$ & $0.0759(18)$ & $0.497(4)$ \\
H13B & 0.488252 & 0.962201 & 1.007970 & $0.091^{*}$ & $0.497(4)$ \\
C14' & $0.6390(15)$ & $0.8437(12)$ & $1.0570(7)$ & $0.079(3)$ & $0.497(4)$ \\
H14B & 0.655646 & 0.893516 & 1.127240 & $0.094^{*}$ & $0.497(4)$ \\
C15' & $0.7133(14)$ & $0.7321(12)$ & $1.0286(5)$ & $0.0843(19)$ & $0.497(4)$ \\
H15B & 0.779040 & 0.702274 & 1.078940 & $0.101^{*}$ & $0.497(4)$ \\
C16' & $0.6923(8)$ & $0.6598(8)$ & $0.9226(5)$ & $0.0793(17)$ & $0.497(4)$ \\
H16B & 0.744737 & 0.582013 & 0.902974 & $0.095^{*}$ & $0.497(4)$ \\
\hline
\end{tabular}

Atomic displacement parameters $\left(\AA^{2}\right)$

\begin{tabular}{lllllll}
\hline & $U^{11}$ & $U^{22}$ & $U^{33}$ & $U^{12}$ & $U^{13}$ & $U^{23}$ \\
\hline Br1 & $0.0842(2)$ & $0.05494(16)$ & $0.05450(16)$ & $-0.00131(12)$ & $0.02403(12)$ & $-0.00748(11)$ \\
S1 & $0.0911(4)$ & $0.0681(4)$ & $0.0419(3)$ & $0.0401(3)$ & $0.0203(3)$ & $0.0126(3)$ \\
F1 & $0.1003(12)$ & $0.1287(15)$ & $0.0746(10)$ & $0.0375(11)$ & $0.0439(9)$ & $0.0375(10)$ \\
N1 & $0.0588(10)$ & $0.0484(10)$ & $0.0428(9)$ & $0.0154(9)$ & $0.0093(8)$ & $0.0067(8)$ \\
C1 & $0.1015(19)$ & $0.0518(13)$ & $0.0448(12)$ & $0.0257(13)$ & $-0.0005(12)$ & $-0.0010(10)$ \\
N2 & $0.0706(12)$ & $0.0494(10)$ & $0.0385(9)$ & $0.0183(9)$ & $0.0088(8)$ & $0.0032(8)$ \\
C2 & $0.145(3)$ & $0.0759(18)$ & $0.0422(13)$ & $0.0652(19)$ & $0.0157(15)$ & $0.0117(12)$ \\
N3 & $0.0696(11)$ & $0.0478(10)$ & $0.0492(10)$ & $0.0162(9)$ & $0.0199(9)$ & $0.0087(8)$ \\
C3 & $0.0593(12)$ & $0.0513(12)$ & $0.0400(10)$ & $0.0240(10)$ & $0.0079(9)$ & $0.0079(9)$ \\
C4 & $0.0643(14)$ & $0.0516(13)$ & $0.0492(12)$ & $0.0108(11)$ & $0.0016(10)$ & $0.0044(10)$ \\
C5 & $0.0504(12)$ & $0.0514(12)$ & $0.0535(12)$ & $0.0119(10)$ & $0.0028(10)$ & $0.0139(10)$ \\
C6 & $0.0733(16)$ & $0.0558(14)$ & $0.0663(15)$ & $0.0074(12)$ & $0.0053(13)$ & $0.0159(12)$ \\
C7 & $0.0708(16)$ & $0.0692(17)$ & $0.0845(19)$ & $0.0101(13)$ & $0.0177(14)$ & $0.0362(16)$ \\
C8 & $0.0585(14)$ & $0.0891(19)$ & $0.0617(15)$ & $0.0311(14)$ & $0.0196(12)$ & $0.0303(14)$ \\
C9 & $0.0575(14)$ & $0.0732(16)$ & $0.0636(15)$ & $0.0204(12)$ & $0.0141(11)$ & $0.0049(12)$ \\
C10 & $0.0515(12)$ & $0.0560(13)$ & $0.0627(14)$ & $0.0092(10)$ & $0.0148(11)$ & $0.0103(11)$ \\
C11 & $0.052(3)$ & $0.043(3)$ & $0.043(3)$ & $0.018(3)$ & $0.015(3)$ & $0.012(2)$ \\
C12 & $0.060(3)$ & $0.084(4)$ & $0.059(4)$ & $0.021(3)$ & $0.014(3)$ & $0.005(3)$ \\
C13 & $0.084(6)$ & $0.101(6)$ & $0.045(4)$ & $0.042(5)$ & $0.018(4)$ & $0.009(4)$ \\
C14 & $0.100(5)$ & $0.083(5)$ & $0.042(3)$ & $0.038(4)$ & $0.006(3)$ & $0.001(3)$ \\
C15 & $0.085(4)$ & $0.096(4)$ & $0.055(3)$ & $0.029(3)$ & $0.003(3)$ & $0.001(3)$ \\
C16 & $0.066(3)$ & $0.077(3)$ & $0.049(3)$ & $0.020(3)$ & $0.011(2)$ & $0.003(2)$ \\
C11' & $0.051(4)$ & $0.053(3)$ & $0.036(3)$ & $0.009(3)$ & $0.016(3)$ & $0.007(2)$ \\
C12 & $0.066(3)$ & $0.058(3)$ & $0.065(3)$ & $0.019(2)$ & $0.009(2)$ & $0.020(2)$ \\
C13' & $0.092(4)$ & $0.057(3)$ & $0.083(4)$ & $0.031(3)$ & $0.041(3)$ & $0.006(3)$ \\
C14' & $0.095(7)$ & $0.073(5)$ & $0.042(4)$ & $0.002(5)$ & $0.013(4)$ & $-0.004(3)$ \\
C15' & $0.087(5)$ & $0.111(7)$ & $0.055(4)$ & $0.037(5)$ & $-0.011(3)$ & $0.012(4)$ \\
C16' & $0.084(4)$ & $0.096(4)$ & $0.072(4)$ & $0.053(4)$ & $0.008(3)$ & $0.002(3)$ \\
& & & & & &
\end{tabular}

Geometric parameters $\left(A,{ }^{o}\right)$

\begin{tabular}{llll}
\hline $\mathrm{S} 1-\mathrm{C} 3$ & $1.720(2)$ & $\mathrm{C} 9-\mathrm{C} 10$ & $1.369(3)$ \\
$\mathrm{S} 1-\mathrm{C} 2$ & $1.848(3)$ & $\mathrm{C} 9-\mathrm{H} 9 \mathrm{~A}$ & 0.9300 \\
$\mathrm{~F} 1-\mathrm{C} 8$ & $1.353(3)$ & $\mathrm{C} 10-\mathrm{H} 10 \mathrm{~A}$ & 0.9300 \\
$\mathrm{~N} 1-\mathrm{C} 4$ & $1.276(3)$ & $\mathrm{C} 11-\mathrm{C} 16$ & $1.353(8)$
\end{tabular}




\begin{tabular}{|c|c|c|c|}
\hline $\mathrm{N} 1-\mathrm{N} 2$ & $1.380(2)$ & $\mathrm{C} 11-\mathrm{C} 12$ & $1.383(7)$ \\
\hline $\mathrm{C} 1-\mathrm{N} 2$ & $1.465(3)$ & $\mathrm{C} 12-\mathrm{C} 13$ & $1.393(10)$ \\
\hline $\mathrm{C} 1-\mathrm{C} 2$ & $1.506(4)$ & $\mathrm{C} 12-\mathrm{H} 12 \mathrm{~A}$ & 0.9300 \\
\hline $\mathrm{C} 1-\mathrm{H} 1 \mathrm{~A}$ & 0.9700 & $\mathrm{C} 13-\mathrm{C} 14$ & $1.364(14)$ \\
\hline $\mathrm{C} 1-\mathrm{H} 1 \mathrm{~B}$ & 0.9700 & $\mathrm{C} 13-\mathrm{H} 13 \mathrm{~A}$ & 0.9300 \\
\hline $\mathrm{N} 2-\mathrm{C} 3$ & $1.339(3)$ & $\mathrm{C} 14-\mathrm{C} 15$ & $1.370(9)$ \\
\hline $\mathrm{C} 2-\mathrm{C} 11^{\prime}$ & $1.505(6)$ & $\mathrm{C} 14-\mathrm{H} 14 \mathrm{~A}$ & 0.9300 \\
\hline $\mathrm{C} 2-\mathrm{C} 11$ & $1.607(7)$ & $\mathrm{C} 15-\mathrm{C} 16$ & $1.370(7)$ \\
\hline $\mathrm{C} 2-\mathrm{H} 2 \mathrm{~A}$ & 0.9800 & $\mathrm{C} 15-\mathrm{H} 15 \mathrm{~A}$ & 0.9300 \\
\hline N3-C3 & $1.297(3)$ & C16-H16A & 0.9300 \\
\hline N3-H3A & 0.9001 & $\mathrm{C} 11^{\prime}-\mathrm{C} 16^{\prime}$ & $1.333(9)$ \\
\hline N3-H3B & 0.9000 & $\mathrm{C} 11^{\prime}-\mathrm{C} 12^{\prime}$ & $1.389(8)$ \\
\hline $\mathrm{C} 4-\mathrm{C} 5$ & $1.458(3)$ & $\mathrm{C} 12^{\prime}-\mathrm{C} 13^{\prime}$ & $1.394(8)$ \\
\hline $\mathrm{C} 4-\mathrm{H} 4 \mathrm{~A}$ & 0.9300 & $\mathrm{C} 12^{\prime}-\mathrm{H} 12 \mathrm{~B}$ & 0.9300 \\
\hline $\mathrm{C} 5-\mathrm{C} 6$ & $1.386(3)$ & $\mathrm{C} 13^{\prime}-\mathrm{C} 14^{\prime}$ & $1.334(12)$ \\
\hline $\mathrm{C} 5-\mathrm{C} 10$ & $1.390(3)$ & $\mathrm{C} 13^{\prime}-\mathrm{H} 13 \mathrm{~B}$ & 0.9300 \\
\hline $\mathrm{C} 6-\mathrm{C} 7$ & $1.379(4)$ & $\mathrm{C} 14^{\prime}-\mathrm{C} 15^{\prime}$ & $1.329(15)$ \\
\hline C6-H6A & 0.9300 & C14'-H14B & 0.9300 \\
\hline $\mathrm{C} 7-\mathrm{C} 8$ & $1.358(4)$ & $\mathrm{C} 15^{\prime}-\mathrm{C} 16^{\prime}$ & $1.399(9)$ \\
\hline C7-H7A & 0.9300 & $\mathrm{C} 15^{\prime}-\mathrm{H} 15 \mathrm{~B}$ & 0.9300 \\
\hline $\mathrm{C} 8-\mathrm{C} 9$ & $1.373(4)$ & $\mathrm{C} 16^{\prime}-\mathrm{H} 16 \mathrm{~B}$ & 0.9300 \\
\hline $\mathrm{C} 3-\mathrm{S} 1-\mathrm{C} 2$ & $90.65(11)$ & $\mathrm{C} 10-\mathrm{C} 9-\mathrm{H} 9 \mathrm{~A}$ & 121.0 \\
\hline $\mathrm{C} 4-\mathrm{N} 1-\mathrm{N} 2$ & $117.98(19)$ & $\mathrm{C} 8-\mathrm{C} 9-\mathrm{H} 9 \mathrm{~A}$ & 121.0 \\
\hline $\mathrm{N} 2-\mathrm{C} 1-\mathrm{C} 2$ & $105.8(2)$ & $\mathrm{C} 9-\mathrm{C} 10-\mathrm{C} 5$ & $121.2(2)$ \\
\hline $\mathrm{N} 2-\mathrm{C} 1-\mathrm{H} 1 \mathrm{~A}$ & 110.6 & $\mathrm{C} 9-\mathrm{C} 10-\mathrm{H} 10 \mathrm{~A}$ & 119.4 \\
\hline $\mathrm{C} 2-\mathrm{C} 1-\mathrm{H} 1 \mathrm{~A}$ & 110.6 & $\mathrm{C} 5-\mathrm{C} 10-\mathrm{H} 10 \mathrm{~A}$ & 119.4 \\
\hline $\mathrm{N} 2-\mathrm{C} 1-\mathrm{H} 1 \mathrm{~B}$ & 110.6 & $\mathrm{C} 16-\mathrm{C} 11-\mathrm{C} 12$ & $118.7(5)$ \\
\hline $\mathrm{C} 2-\mathrm{C} 1-\mathrm{H} 1 \mathrm{~B}$ & 110.6 & $\mathrm{C} 16-\mathrm{C} 11-\mathrm{C} 2$ & $133.2(5)$ \\
\hline $\mathrm{H} 1 \mathrm{~A}-\mathrm{C} 1-\mathrm{H} 1 \mathrm{~B}$ & 108.7 & $\mathrm{C} 12-\mathrm{C} 11-\mathrm{C} 2$ & $108.2(5)$ \\
\hline $\mathrm{C} 3-\mathrm{N} 2-\mathrm{N} 1$ & $116.37(17)$ & $\mathrm{C} 11-\mathrm{C} 12-\mathrm{C} 13$ & $120.0(6)$ \\
\hline $\mathrm{C} 3-\mathrm{N} 2-\mathrm{C} 1$ & $115.67(18)$ & $\mathrm{C} 11-\mathrm{C} 12-\mathrm{H} 12 \mathrm{~A}$ & 120.0 \\
\hline $\mathrm{N} 1-\mathrm{N} 2-\mathrm{C} 1$ & $127.52(19)$ & $\mathrm{C} 13-\mathrm{C} 12-\mathrm{H} 12 \mathrm{~A}$ & 120.0 \\
\hline $\mathrm{C} 11^{\prime}-\mathrm{C} 2-\mathrm{C} 1$ & $129.4(4)$ & $\mathrm{C} 14-\mathrm{C} 13-\mathrm{C} 12$ & $120.3(7)$ \\
\hline $\mathrm{C} 1-\mathrm{C} 2-\mathrm{C} 11$ & $104.7(3)$ & $\mathrm{C} 14-\mathrm{C} 13-\mathrm{H} 13 \mathrm{~A}$ & 119.8 \\
\hline $\mathrm{C} 11^{\prime}-\mathrm{C} 2-\mathrm{S} 1$ & $104.9(3)$ & $\mathrm{C} 12-\mathrm{C} 13-\mathrm{H} 13 \mathrm{~A}$ & 119.8 \\
\hline $\mathrm{C} 1-\mathrm{C} 2-\mathrm{S} 1$ & $105.04(17)$ & $\mathrm{C} 13-\mathrm{C} 14-\mathrm{C} 15$ & $119.0(6)$ \\
\hline $\mathrm{C} 11-\mathrm{C} 2-\mathrm{S} 1$ & $112.7(2)$ & $\mathrm{C} 13-\mathrm{C} 14-\mathrm{H} 14 \mathrm{~A}$ & 120.5 \\
\hline $\mathrm{C} 1-\mathrm{C} 2-\mathrm{H} 2 \mathrm{~A}$ & 111.4 & $\mathrm{C} 15-\mathrm{C} 14-\mathrm{H} 14 \mathrm{~A}$ & 120.5 \\
\hline $\mathrm{C} 11-\mathrm{C} 2-\mathrm{H} 2 \mathrm{~A}$ & 111.4 & $\mathrm{C} 16-\mathrm{C} 15-\mathrm{C} 14$ & $120.5(6)$ \\
\hline $\mathrm{S} 1-\mathrm{C} 2-\mathrm{H} 2 \mathrm{~A}$ & 111.4 & $\mathrm{C} 16-\mathrm{C} 15-\mathrm{H} 15 \mathrm{~A}$ & 119.7 \\
\hline $\mathrm{C} 3-\mathrm{N} 3-\mathrm{H} 3 \mathrm{~A}$ & 117.3 & $\mathrm{C} 14-\mathrm{C} 15-\mathrm{H} 15 \mathrm{~A}$ & 119.7 \\
\hline $\mathrm{C} 3-\mathrm{N} 3-\mathrm{H} 3 \mathrm{~B}$ & 119.6 & $\mathrm{C} 11-\mathrm{C} 16-\mathrm{C} 15$ & $121.5(5)$ \\
\hline $\mathrm{H} 3 \mathrm{~A}-\mathrm{N} 3-\mathrm{H} 3 \mathrm{~B}$ & 120.5 & $\mathrm{C} 11-\mathrm{C} 16-\mathrm{H} 16 \mathrm{~A}$ & 119.2 \\
\hline $\mathrm{N} 3-\mathrm{C} 3-\mathrm{N} 2$ & $123.59(19)$ & $\mathrm{C} 15-\mathrm{C} 16-\mathrm{H} 16 \mathrm{~A}$ & 119.2 \\
\hline $\mathrm{N} 3-\mathrm{C} 3-\mathrm{S} 1$ & $123.18(17)$ & $\mathrm{C} 16^{\prime}-\mathrm{C} 11^{\prime}-\mathrm{C} 12^{\prime}$ & $118.8(6)$ \\
\hline $\mathrm{N} 2-\mathrm{C} 3-\mathrm{S} 1$ & $113.23(16)$ & $\mathrm{C} 16^{\prime}-\mathrm{C} 11^{\prime}-\mathrm{C} 2$ & $119.7(5)$ \\
\hline $\mathrm{N} 1-\mathrm{C} 4-\mathrm{C} 5$ & $120.0(2)$ & $\mathrm{C} 12^{\prime}-\mathrm{C} 11^{\prime}-\mathrm{C} 2$ & $121.2(5)$ \\
\hline
\end{tabular}




\begin{tabular}{|c|c|}
\hline $\mathrm{N} 1-\mathrm{C} 4-\mathrm{H} 4 \mathrm{~A}$ & 120.0 \\
\hline $\mathrm{C} 5-\mathrm{C} 4-\mathrm{H} 4 \mathrm{~A}$ & 120.0 \\
\hline $\mathrm{C} 6-\mathrm{C} 5-\mathrm{C} 10$ & $118.6(2)$ \\
\hline $\mathrm{C} 6-\mathrm{C} 5-\mathrm{C} 4$ & $119.1(2)$ \\
\hline $\mathrm{C} 10-\mathrm{C} 5-\mathrm{C} 4$ & $122.3(2)$ \\
\hline $\mathrm{C} 7-\mathrm{C} 6-\mathrm{C} 5$ & $120.8(3)$ \\
\hline $\mathrm{C} 7-\mathrm{C} 6-\mathrm{H} 6 \mathrm{~A}$ & 119.6 \\
\hline $\mathrm{C} 5-\mathrm{C} 6-\mathrm{H} 6 \mathrm{~A}$ & 119.6 \\
\hline $\mathrm{C} 8-\mathrm{C} 7-\mathrm{C} 6$ & $118.3(2)$ \\
\hline $\mathrm{C} 8-\mathrm{C} 7-\mathrm{H} 7 \mathrm{~A}$ & 120.9 \\
\hline $\mathrm{C} 6-\mathrm{C} 7-\mathrm{H} 7 \mathrm{~A}$ & 120.9 \\
\hline $\mathrm{F} 1-\mathrm{C} 8-\mathrm{C} 7$ & $119.0(2)$ \\
\hline $\mathrm{F} 1-\mathrm{C} 8-\mathrm{C} 9$ & $117.9(3)$ \\
\hline $\mathrm{C} 7-\mathrm{C} 8-\mathrm{C} 9$ & $123.1(2)$ \\
\hline $\mathrm{C} 10-\mathrm{C} 9-\mathrm{C} 8$ & $118.0(3)$ \\
\hline $\mathrm{C} 4-\mathrm{N} 1-\mathrm{N} 2-\mathrm{C} 3$ & $-169.1(2)$ \\
\hline $\mathrm{C} 4-\mathrm{N} 1-\mathrm{N} 2-\mathrm{C} 1$ & $3.0(3)$ \\
\hline $\mathrm{C} 2-\mathrm{C} 1-\mathrm{N} 2-\mathrm{C} 3$ & $-26.1(3)$ \\
\hline $\mathrm{C} 2-\mathrm{C} 1-\mathrm{N} 2-\mathrm{N} 1$ & $161.8(2)$ \\
\hline $\mathrm{N} 2-\mathrm{C} 1-\mathrm{C} 2-\mathrm{C} 11^{\prime}$ & $155.8(4)$ \\
\hline $\mathrm{N} 2-\mathrm{C} 1-\mathrm{C} 2-\mathrm{C} 11$ & $150.2(3)$ \\
\hline $\mathrm{N} 2-\mathrm{C} 1-\mathrm{C} 2-\mathrm{S} 1$ & $31.4(2)$ \\
\hline $\mathrm{C} 3-\mathrm{S} 1-\mathrm{C} 2-\mathrm{C} 11^{\prime}$ & $-163.6(3)$ \\
\hline $\mathrm{C} 3-\mathrm{S} 1-\mathrm{C} 2-\mathrm{C} 1$ & $-24.92(19)$ \\
\hline $\mathrm{C} 3-\mathrm{S} 1-\mathrm{C} 2-\mathrm{C} 11$ & $-138.3(3)$ \\
\hline $\mathrm{N} 1-\mathrm{N} 2-\mathrm{C} 3-\mathrm{N} 3$ & $-0.7(3)$ \\
\hline $\mathrm{C} 1-\mathrm{N} 2-\mathrm{C} 3-\mathrm{N} 3$ & $-173.7(2)$ \\
\hline $\mathrm{N} 1-\mathrm{N} 2-\mathrm{C} 3-\mathrm{S} 1$ & $179.87(14)$ \\
\hline $\mathrm{C} 1-\mathrm{N} 2-\mathrm{C} 3-\mathrm{S} 1$ & $6.8(3)$ \\
\hline $\mathrm{C} 2-\mathrm{S} 1-\mathrm{C} 3-\mathrm{N} 3$ & $-168.1(2)$ \\
\hline $\mathrm{C} 2-\mathrm{S} 1-\mathrm{C} 3-\mathrm{N} 2$ & $11.35(19)$ \\
\hline $\mathrm{N} 2-\mathrm{N} 1-\mathrm{C} 4-\mathrm{C} 5$ & $-177.36(19)$ \\
\hline $\mathrm{N} 1-\mathrm{C} 4-\mathrm{C} 5-\mathrm{C} 6$ & $174.4(2)$ \\
\hline $\mathrm{N} 1-\mathrm{C} 4-\mathrm{C} 5-\mathrm{C} 10$ & $-4.2(4)$ \\
\hline $\mathrm{C} 10-\mathrm{C} 5-\mathrm{C} 6-\mathrm{C} 7$ & $0.8(4)$ \\
\hline $\mathrm{C} 4-\mathrm{C} 5-\mathrm{C} 6-\mathrm{C} 7$ & $-177.9(2)$ \\
\hline $\mathrm{C} 5-\mathrm{C} 6-\mathrm{C} 7-\mathrm{C} 8$ & $1.2(4)$ \\
\hline $\mathrm{C} 6-\mathrm{C} 7-\mathrm{C} 8-\mathrm{F} 1$ & $179.3(2)$ \\
\hline $\mathrm{C} 6-\mathrm{C} 7-\mathrm{C} 8-\mathrm{C} 9$ & $-2.3(4)$ \\
\hline $\mathrm{F} 1-\mathrm{C} 8-\mathrm{C} 9-\mathrm{C} 10$ & $179.6(2)$ \\
\hline $\mathrm{C} 7-\mathrm{C} 8-\mathrm{C} 9-\mathrm{C} 10$ & $1.1(4)$ \\
\hline $\mathrm{C} 8-\mathrm{C} 9-\mathrm{C} 10-\mathrm{C} 5$ & $1.0(4)$ \\
\hline
\end{tabular}

$\mathrm{C} 11^{\prime}-\mathrm{C} 12^{\prime}-\mathrm{C} 13^{\prime}$

$\mathrm{C} 11^{\prime}-\mathrm{C} 12^{\prime}-\mathrm{H} 12 \mathrm{~B}$

$\mathrm{C} 13^{\prime}-\mathrm{C} 12^{\prime}-\mathrm{H} 12 \mathrm{~B}$

C14'-C13'-C12'

$\mathrm{C} 14^{\prime}-\mathrm{C} 13^{\prime}-\mathrm{H} 13 \mathrm{~B}$

$\mathrm{C} 12^{\prime}-\mathrm{C} 13^{\prime}-\mathrm{H} 13 \mathrm{~B}$

$\mathrm{C} 15^{\prime}-\mathrm{C} 14^{\prime}-\mathrm{C} 13^{\prime}$

C15'-C14'-H14B

C13'-C14'-H14B

C14'-C15'-C16'

$\mathrm{C} 14^{\prime}-\mathrm{C} 15^{\prime}-\mathrm{H} 15 \mathrm{~B}$

$\mathrm{C} 16^{\prime}-\mathrm{C} 15^{\prime}-\mathrm{H} 15 \mathrm{~B}$

$\mathrm{C} 11^{\prime}-\mathrm{C} 16^{\prime}-\mathrm{C} 15^{\prime}$

C11'-C16'-H16B

$\mathrm{C} 15^{\prime}-\mathrm{C} 16^{\prime}-\mathrm{H} 16 \mathrm{~B}$

$\mathrm{C} 6-\mathrm{C} 5-\mathrm{C} 10-\mathrm{C} 9$

$\mathrm{C} 4-\mathrm{C} 5-\mathrm{C} 10-\mathrm{C} 9$

$\mathrm{C} 1-\mathrm{C} 2-\mathrm{C} 11-\mathrm{C} 16$

$\mathrm{S} 1-\mathrm{C} 2-\mathrm{C} 11-\mathrm{C} 16$

$\mathrm{C} 1-\mathrm{C} 2-\mathrm{C} 11-\mathrm{C} 12$

$\mathrm{S} 1-\mathrm{C} 2-\mathrm{C} 11-\mathrm{C} 12$

$\mathrm{C} 16-\mathrm{C} 11-\mathrm{C} 12-\mathrm{C} 13$

$\mathrm{C} 2-\mathrm{C} 11-\mathrm{C} 12-\mathrm{C} 13$

$\mathrm{C} 11-\mathrm{C} 12-\mathrm{C} 13-\mathrm{C} 14$

$\mathrm{C} 12-\mathrm{C} 13-\mathrm{C} 14-\mathrm{C} 15$

$\mathrm{C} 13-\mathrm{C} 14-\mathrm{C} 15-\mathrm{C} 16$

$\mathrm{C} 12-\mathrm{C} 11-\mathrm{C} 16-\mathrm{C} 15$

$\mathrm{C} 2-\mathrm{C} 11-\mathrm{C} 16-\mathrm{C} 15$

$\mathrm{C} 14-\mathrm{C} 15-\mathrm{C} 16-\mathrm{C} 11$

$\mathrm{C} 1-\mathrm{C} 2-\mathrm{C} 11^{\prime}-\mathrm{C} 16^{\prime}$

$\mathrm{S} 1-\mathrm{C} 2-\mathrm{C} 11^{\prime}-\mathrm{C} 16^{\prime}$

$\mathrm{C} 1-\mathrm{C} 2-\mathrm{C} 11^{\prime}-\mathrm{C} 12^{\prime}$

$\mathrm{S} 1-\mathrm{C} 2-\mathrm{C} 11^{\prime}-\mathrm{C} 12^{\prime}$

$\mathrm{C} 16^{\prime}-\mathrm{C} 11^{\prime}-\mathrm{C} 12^{\prime}-\mathrm{C} 13^{\prime}$

$\mathrm{C} 2-\mathrm{C} 11^{\prime}-\mathrm{C} 12^{\prime}-\mathrm{C} 13^{\prime}$

$\mathrm{C} 11^{\prime}-\mathrm{C} 12^{\prime}-\mathrm{C} 13^{\prime}-\mathrm{C} 14^{\prime}$

$\mathrm{C} 12^{\prime}-\mathrm{C} 13^{\prime}-\mathrm{C} 14^{\prime}-\mathrm{C} 15^{\prime}$

$\mathrm{C} 13^{\prime}-\mathrm{C} 14^{\prime}-\mathrm{C} 15^{\prime}-\mathrm{C} 16^{\prime}$

$\mathrm{C} 12^{\prime}-\mathrm{C} 11^{\prime}-\mathrm{C} 16^{\prime}-\mathrm{C} 15^{\prime}$

$\mathrm{C} 2-\mathrm{C} 11^{\prime}-\mathrm{C} 16^{\prime}-\mathrm{C} 15^{\prime}$

$\mathrm{C} 14^{\prime}-\mathrm{C} 15^{\prime}-\mathrm{C} 16^{\prime}-\mathrm{C} 11^{\prime}$
$119.1(5)$

120.4

120.4

$120.3(6)$

119.9

119.9

$121.0(8)$

119.5

119.5

119.7 (8)

120.1

120.1

$121.0(6)$

119.5

119.5

$-2.0(4)$

$176.7(2)$

$-1.1(7)$

$112.5(6)$

$-179.8(4)$

$-66.2(5)$

$0.1(10)$

$178.9(6)$

$0.8(14)$

$-1.5(15)$

$1.4(12)$

$-0.2(9)$

$-178.7(5)$

$-0.6(10)$

$-64.5(7)$

$59.9(7)$

$109.3(6)$

$-126.3(5)$

$2.2(9)$

$-171.6(5)$

$-0.9(9)$

$-1.0(13)$

$1.5(16)$

$-1.7(11)$

$172.2(6)$

$-0.2(14)$

Hydrogen-bond geometry $\left(A,{ }^{\circ}\right)$

\begin{tabular}{lllll}
\hline$D-\mathrm{H} \cdots A$ & $D-\mathrm{H}$ & $\mathrm{H} \cdots A$ & $D \cdots A$ & $D-\mathrm{H} \cdots A$ \\
\hline $\mathrm{N} 3-\mathrm{H} 3 A \cdots \mathrm{Br} 1$ & 0.90 & 2.36 & $3.2557(18)$ & 172
\end{tabular}


supporting information

$\mathrm{N} 3-\mathrm{H} 3 B \cdots \mathrm{Br} 1^{\mathrm{i}}$

0.90

2.55

$3.3552(18)$

150

$\mathrm{C} 4-\mathrm{H} 4 A \cdots \mathrm{Br} 1^{\mathrm{ii}}$

0.93

2.99

$3.726(2)$

137

Symmetry codes: (i) $-x+1,-y,-z+1$; (ii) $x+1, y+1, z$. 